\title{
Reality or Locality? Proposed Test to Decide How Nature Breaks Bell's Inequality
}

\author{
Johan Hansson \\ Department of Physics, Luleå University of Technology, 97187 Luleå, Sweden \\ Correspondence should be addressed to Johan Hansson, c.johan.hansson@ltu.se
}

Received 17 August 2011; Revised 27 October 2011; Accepted 27 October 2011

Academic Editor: Ali Hussain Reshak

Copyright () 2012 Johan Hansson. This is an open access article distributed under the Creative Commons Attribution License, which permits unrestricted use, distribution, and reproduction in any medium, provided the original work is properly cited.

Bell's theorem, and its experimental tests, has shown that the two premises for Bell's inequality-locality and objective realitycannot both hold in nature, as Bell's inequality is broken. A simple test is proposed, which for the first time may decide which alternative nature actually prefers on the fundamental, quantum level. If each microscopic event is truly random (e.g., as assumed in orthodox quantum mechanics) objective reality is not valid whereas if each event is described by an unknown but deterministic mechanism ("hidden variables") locality is not valid. This may be analyzed and decided by the well-known reconstruction method of Ruelle and Takens; in the former case no structure should be discerned, in the latter a reconstructed structure should be visible. This could in principle be tested by comparing individual "hits" in a double-slit experiment, but in practice a single fluorescent atom, and its (seemingly random) temporal switching between active/inactive states would possibly be better/more practical, easier to set up, observe, and analyze. However, only imagination limits the list of possible experimental setups.

Through Bell's theorem [1, 2], which put the (in)famous Einstein-Podolsky-Rosen [3] argument on a solid and testable footing, and experimental tests thereof [4-8] it has been proven beyond reasonable doubt that no "locally realistic" fundamental model of the world can be correct. That is, a "sensible" worldview, such as that proposed in [3], is unfortunately untenable.

So either the objective reality-condition (that things exist in definite states whether we look or not) must be broken, for example, as in orthodox quantum mechanics, or the localitycondition (that events arbitrarily far away cannot affect what happens here and now-relativistic separability and causality) must be broken, for example, as in nonlocal hidden variable theories. The variables are called "hidden" because their existence is only conjectured and beyond our (present) control, but meant to complete quantum mechanics into a uniform description of micro and macro (Bell himself was heavily biased towards a hidden variable resolution of the problem [9]). The first detailed such theory, perfectly deterministic and compatible with all known experimental data, was [10]. Notice, however, that we are not necessarily considering any specific existing hidden variable theory, but an "ultimate" hidden variable theory that in principle decides everything deterministically. In contrast in the orthodox approach to quantum mechanics the quantum particles in effect behave as particles when observed and as waves when not observed-thereby, and at the most fundamental level, introducing the ill-defined act of observation ("measurement problem," "collapse of the wave function," and Bohr's "irreversible act of measurement"), whereas particles in hidden variable theories always behave as particles but are being "pushed around" by the underlying (hidden) dynamics. In such deterministic systems the present state completely and uniquely determines the future, but as is well-known chaotic systems can "impersonate" randomness due to their extreme sensitivity to initial conditions; in a nutshell chaos is about order and disorder in deterministic systems that are nonlinear.

So far, it has not been possible to distinguish between the locality versus reality alternatives, and the choice has been mainly one of personal taste.

However, as hidden variable theories are deterministic (quantum particles behaving as realistic classical particles all the time, encoding Einstein's "elements of physical reality" [3]) and orthodox quantum mechanics fundamentally probabilistic (each individual event/measurement assumed 


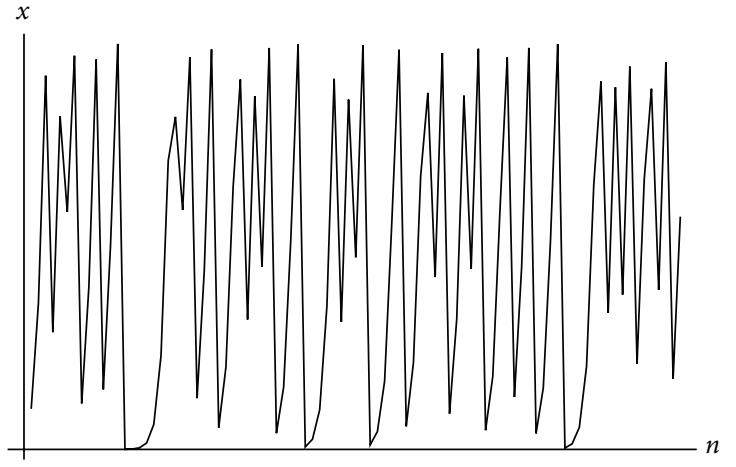

FIGURE 1: Seemingly random data, actually generated by the simple and deterministic "logistic mapping" in its chaotic region, see text, and [13].

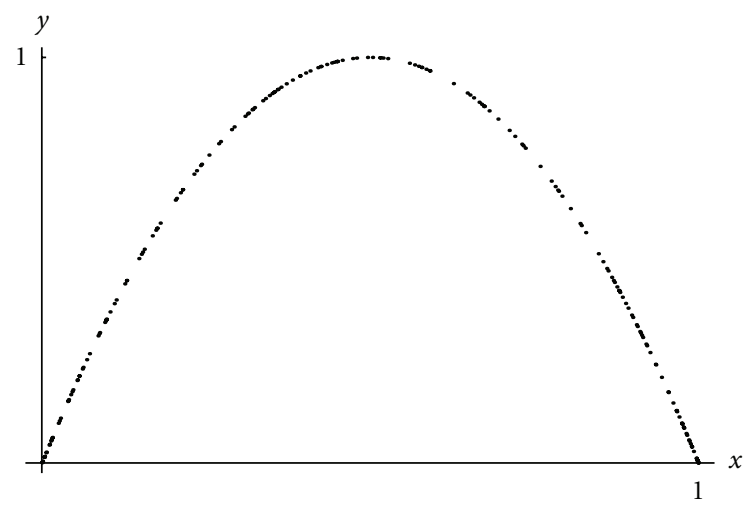

Figure 2: The reconstructed attractor in 2D from the data in Figure 1, using (1), showing that its "randomness" has its origin in dynamical deterministic chaos.

to be completely random), it should be possible to experimentally test the distinction between them.

An experiment to test this possibility could be devised in analogy to the confirmation of deterministic chaos in a dripping water faucet $[11,12]$. It is of course wellknown that deterministic chaos requires nonlinear systems whereas the Schrödinger equation is linear. However, most hidden variable theories like the original by Bohm [10] are manifestly nonlinear (As an aside, if hidden variables is the correct way to explain the violation of Bell's inequality this could make true quantum chaos possible, as opposed to the usual notion of "quantum chaos" which is concerned with quantum signatures of corresponding systems known to be chaotic in the classical case, as the linear structure of the Schrödinger equation alone does not support true chaos).

If we, for example, replace the dripping faucet with a double-slit experiment (According to R. P. Feynman the double-slit experiment "... has in it the heart of quantum mechanics. In reality, it contains the only mystery.", The Feynman Lectures on Physics, Vol.III, p. 1-1) with individual quantum entities (electrons, neutrons, photons, etc.), the effectively one-dimensional position $\left(q_{i}\right)$ of the successive "hits" on the detector screen, in effect defining a discrete

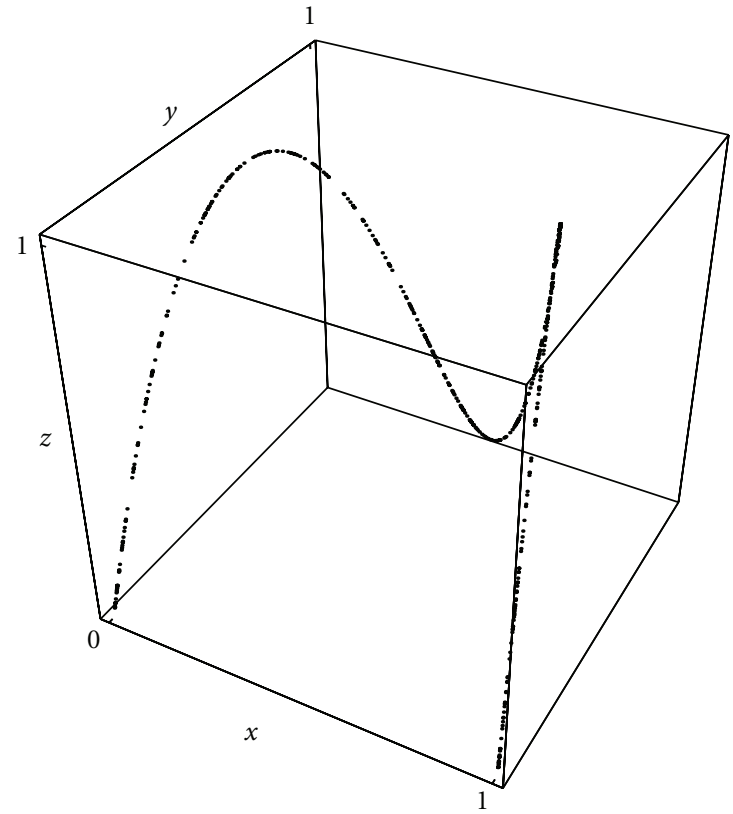

Figure 3: Reconstructed attractor in 3D from the data in Figure 1, again using (1).

time series, can be used to try to reconstruct a chaotic attractor, in case the underlying theory is dissipative, or a deterministic structure in phase space, in case it is nondissipative (Hamiltonian), by applying a method [14, 15] of converting a single data series into a phase space portrait via "delay coordinate embedding." This can be accomplished, assuming a suitably low-dimensional attractor/structure, by defining the coordinates as follows:

$$
x=q_{i}, \quad y=q_{i+1}, \quad z=q_{i+2} .
$$

A given $i$ then gives a point, $(x, y, z)$, in phase space.

To give an elementary example, the seemingly random data in Figure 1 is really due to the deceptively simple, but actually incredibly rich, "logistic mapping,"

$$
x_{n+1}=k x_{n}\left(1-x_{n}\right),
$$

in its highly chaotic regime with $k=4$ [13].

The reconstructed attractor, using the method described above, is seen in Figure 2 (2D) and in Figure 3 (3D).

We do not, however, expect that an eventual attractor/structure in real quantum mechanical data will be so simple and low dimensional, even though the logistic mapping has been shown to be in qualitative and quantitative agreement with numerous real-life systems in all branches of science, see, for example, [16] for some early examples. This would be very surprising if not for the remarkable fact that there exists a "universality" in this kind of chaos [17].

In a sense, the logistic mapping is just like a model for observing "random" hits on an effectively 1D-detector screen of unit length (arbitrarily defined), just like in the double-slit experiment. The detector in effect defines a natural Poincaré section—a discrete "stroboscope" mapping 


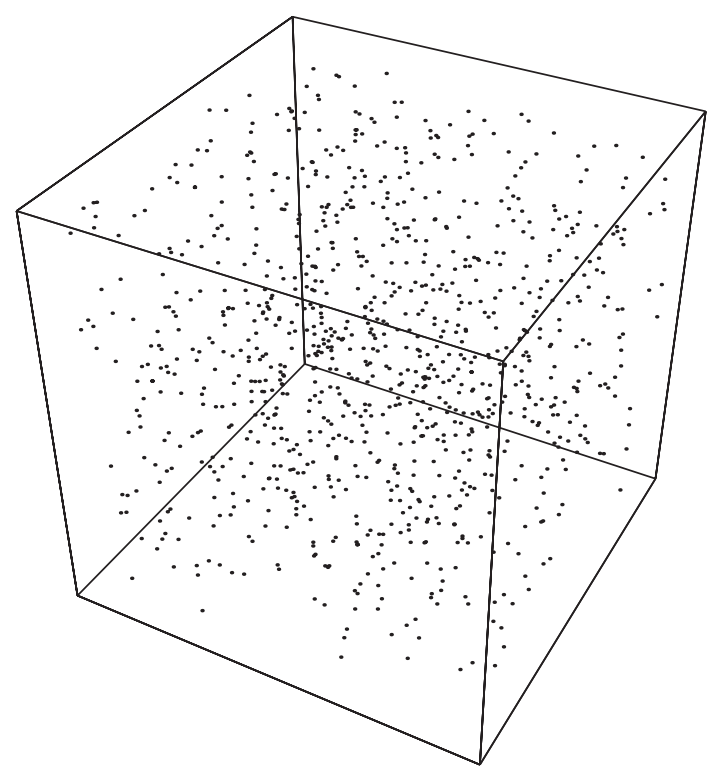

FIgUre 4: When no dynamical relation between the data points $\left(q_{i}\right)$ exists, no structure is obtained by the reconstruction mechanism, (1). This would be the case for "orthodox" quantum mechanics where each individual hit/result/event is assumed to be completely random. The world could then not be objectively real, but could be local.

of the unit interval onto itself - of the underlying continuous dynamics described by differential equations. If there is a deterministic mechanism underlying the "random" hits on the screen, creating the known statistical distribution after many hits, it should then show up as a structure in reconstructed phase space.

In principle, to capture all emitted quantum particles, the ideal would be to have a perfectly efficient $4 \pi$-detector, faithfully recording each individual quantum particle on its "latitude and longitude." A 2D-iterated mapping, of the classic predator-prey kind, would then be a model for the successive hits, the simplest one using "nonoverlapping generations," where each hit is described by two coordinates (originally the populations of predator and prey species) and is determined by the previous hit through a mapping of the form

$$
x_{n+1}=f\left(x_{n}, y_{n}\right), \quad y_{n+1}=g\left(x_{y}, y_{n}\right) .
$$

One such model, the Hénon mapping [18]

$$
x_{n+1}=y_{n}+1-a x_{n}^{2}, \quad y_{n+1}=b x_{n},
$$

gives the famous Hénon-attractor. For the canonical values $a=1.4$ and $b=0.3$ the Hénon map is chaotic; each individual hit appears random, but a clear structure builds up over time, analogous to hits in the double-slit experiment. In the former case the structure is fractal [19] whereas in the latter case it may or may not be.

However, one could argue that any eventual hidden variables must "know" that we have restricted the "landing platform" for the quantum particle to an effectively $1 \mathrm{D}$

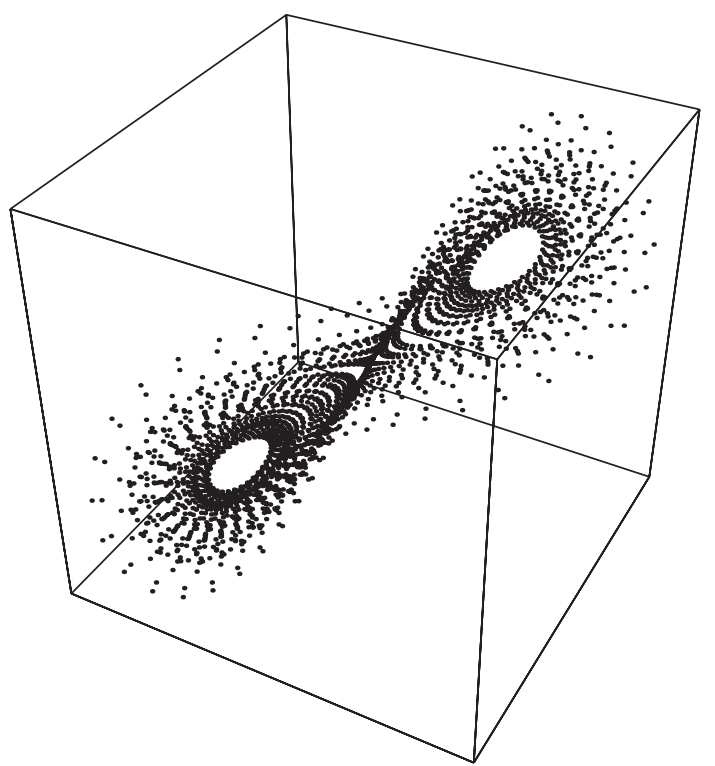

FIGURE 5: If "hidden variables" are governed and determined by dissipative equations an attractor will be reconstructed by the $q_{i}$ s. To mimic the apparently random behavior of quantum mechanical data it will be a "strange attractor" with noninteger (fractal [19]) dimension analogous to the famous Lorenz-attractor, here reconstructed from time series data from only one of the three variables of the Lorenz system [20] — the very first concrete example of dissipative chaos. Any apparent attractor structure would tell us nature is not local-causes arbitrarily far may affect results "here" - that is, there are influences going faster than light (even if we cannot control them for practical telegraphy). Furthermore, it would indicate that the orthodox ("Copenhagen") interpretation of quantum mechanics is wrong.

strip, so that additional spatial variables are superfluous. The hidden variables must also keep track of if one or both slits are open and relay that information nonlocally (faster than the speed of light) to the detector screen, as in [10], to comply with the violation of Bell's inequality.

As modern technology has made it possible to trap and observe individual quantum objects, such as atoms, it might be better and easier to exploit this fact than trying to use the mythical double-slit. Measurements of "quantum jumps" in single atoms $[23,24]$, and the resulting fluctuation of their fluorescent on/off states, may make an ideal testing ground where recorded data should already be present (the time series underlying Figure 2 in both articles [23, 24] could in principle be directly inserted into (1) above), but only imagination limits the list of possible experimental setups.

If the seemingly random florescence gives rise to a distinct structure in phase space, with noninteger fractal dimension, onto which the phase space points are concentrated, it would be a clear indication that it is actually the consequence of dynamical deterministic chaos (i.e., hidden variables), in direct analogy to how $[11,12]$ revealed deterministic chaos in the dynamics of the dripping water faucet (Figure 6). For examples of qualitatively typical chaotic attractors/structures see, for example, the figures in $[11,12]$ or the famous examples presented in the figures in this article 


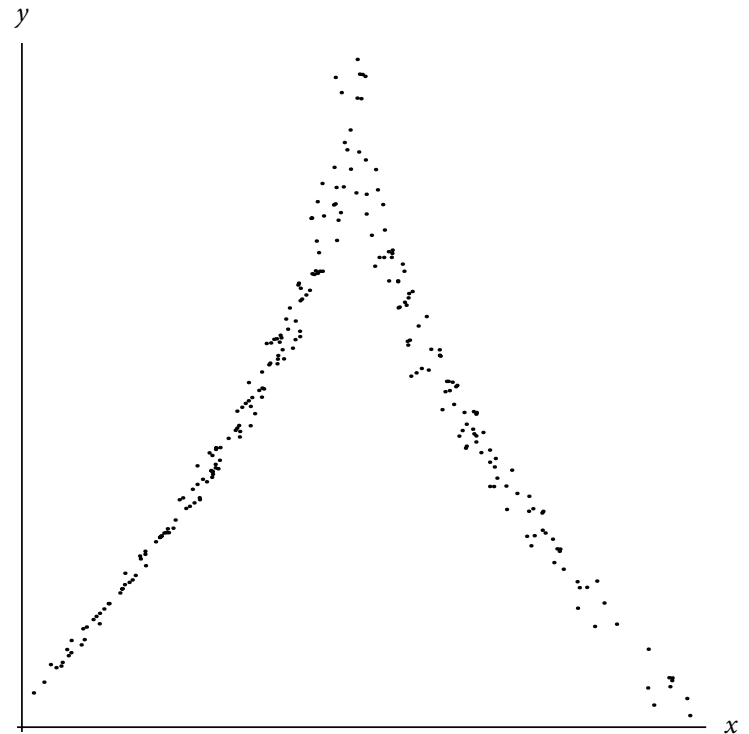

Figure 6: "The Lorenz map"-when successive, erratically fluctuating, amplitude maxima were plotted for the Lorenz attractor (previous figure) using a technique analogous to the one described in this article, the surprising result was this nearly one-dimensional attractor; hidden order in chaos [20], and a concrete simple example of the relation between continuous dynamics and discrete mappings.

(accompanied by their respective physical implications to the problem at hand in the figure captions to Figures 5 and 7). However, the exact shape, dimension, and complexity will be governed by the (unknown) detailed underlying dynamics. The rest of the analysis carries through just like in $[11,12]$.

In fact, in the present case it is in principle even easier to obtain a conclusive result as any observed structure indicates a deviation from the usual assumption of total randomness of quantum mechanics - where it is normally assumed that, for example, the hit of an individual particle is a completely independent and truly random process-even if one has collected one million successive data points the next one, according to orthodox quantum mechanics, will be a complete surprise and impossible to predict even in principle, see Figure 4.

So, in a perfect world it should be easy to potentially disprove orthodox quantum mechanics. A practical problem is of course that there exist no perfect particle detectors, which results in missing part of the series and also in the introduction of noise in the data. The more of the series one misses, the harder it becomes to reconstruct an (eventual) attractor/structure. This may, as stated above, be circumvented by observing, for example, single atoms exhibiting quantum jumps as this "... can be detected with unity quantum efficiency" [23].

If, however, no attractor/structure is found in the experimental data, that is, if the points are scattered randomly in phase space, as in Figure 4, where every $q_{i}$ has been generated at random, then quantum mechanical "measurements" (e.g., hits on detector screen, timing between on/off states, etc.)

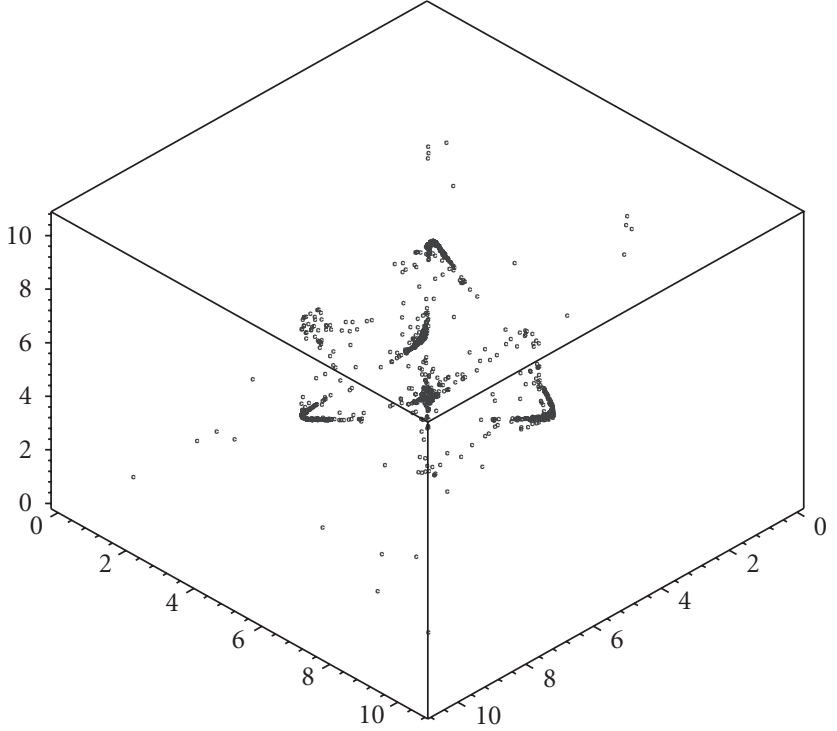

Figure 7: If "hidden variables" are governed and determined by nondissipative (Hamiltonian) equations no attractor will result, yet a structure differing from pure randomness will emerge. Such a result would imply the same conclusion regarding the world as noted in the text accompanying Figure 5. The example shows the modern reconstructed phase space of the "restricted circular threebody problem" in astronomy [21], where Poincaré first glimpsed what today is known as deterministic chaos, nondissipative in this case. This (the corrected and printed version [22]) was his winning contribution (price money: 2,500 Swedish Kronor) to a contest announced in 1885 to celebrate the 60th birthday of the Swedish King Oscar II in 1889. What Poincaré found was that small changes in the initial conditions (such as positions and initial velocities of planets) produced huge and unpredictable outcomes-deterministic chaos in today's parlance.

probably cannot be described by deterministic equations, and some truly stochastic effect(s) must instead be at work, for example, as assumed in orthodox quantum mechanics.

Hence, it should be possible to test, and potentially falsify: either the hypothesis that quantum randomness is due to underlying deterministic dynamics-hidden variables (in which case the "randomness" actually would merely be apparent, not fundamental)_-without having to know and penetrate the details of the underlying equations, or the standard fundamentally probabilistic interpretation/postulate of Born as used in orthodox quantum mechanics, and hence answer if nature prefers to break locality or objective reality on her fundamental level. In case of the former, it would indicate an unexpected and deep hidden connection between the three great revolutions of 20th-century science; relativity, quantum mechanics, and chaos theory, and perhaps even point the way towards a unified complex nonlinear systems theory of the future.

\section{References}

[1] J. S. Bell, "On the Einstein Podolsky Rosen paradox," Physics, vol. 1, no. 3, p. 195, 1964. 
[2] J. F. Clauser, M. A. Horne, A. Shimony, and R. A. Holt, "Proposed experiment to test local hidden-variable theories," Physical Review Letters, vol. 23, no. 15, pp. 880-884, 1969.

[3] A. Einstein, B. Podolsky, and N. Rosen, "Can quantummechanical description of physical reality be considered complete?" Physical Review, vol. 47, no. 10, pp. 777-780, 1935.

[4] S. J. Freedman and J. F. Clauser, "Experimental test of local hidden-variable theories," Physical Review Letters, vol. 28, no. 14, pp. 938-941, 1972.

[5] A. Aspect, P. Grangier, and G. Roger, "Experimental realization of Einstein-Podolsky-Rosen-Bohm Gedankenexperiment: a new violation of Bell's inequalities," Physical Review Letters, vol. 49, no. 2, pp. 91-94, 1982.

[6] A. Aspect, J. Dalibard, and G. Roger, "Experimental test of Bell's inequalities using time- varying analyzers," Physical Review Letters, vol. 49, no. 25, pp. 1804-1807, 1982.

[7] W. Tittel, J. Brendel, H. Zbinden, and N. Gisin, "Violation of Bell inequalities by photons more than $10 \mathrm{~km}$ apart," Physical Review Letters, vol. 81, no. 17, pp. 3563-3566, 1998.

[8] G. Weihs, T. Jennewein, C. Simon, H. Weinfurter, and A. Zeilinger, "Violation of Bell's inequality under strict einstein locality conditions," Physical Review Letters, vol. 81, no. 23, pp. 5039-5042, 1998.

[9] J. S. Bell, Speakable and Unspeakable in Quantum Mechanics, Cambridge University Press, Cambridge, UK, 2nd edition, 2004.

[10] D. Bohm, "A suggested interpretation of the quantum theory in terms of "hidden" variables. II," Physical Review, vol. 85, no. 2, pp. 180-193, 1952.

[11] R. Shaw, The Dripping Faucet as a Model Chaotic System, Aerial Press, Santa Cruz, Calif, USA, 1984.

[12] P. Martien, S. C. Pope, P. L. Scott, and R. S. Shaw, "The chaotic behavior of the leaky faucet," Physics Letters A, vol. 110, no. 7-8, pp. 399-404, 1985.

[13] R. M. May, "Simple mathematical models with very complicated dynamics," Nature, vol. 261, no. 5560, pp. 459-467, 1976.

[14] N. H. Packard, J. P. Crutchfield, J. D. Farmer, and R. S. Shaw, "Geometry from a time series," Physical Review Letters, vol. 45, no. 9, pp. 712-716, 1980.

[15] F. Takens, "Detecting strange attractors in turbulence," in Dynamical Systems and Turbulence, D. A. Rand and L. S. Young, Eds., vol. 898 of Lecture Notes in Mathematics, pp. 367381, Springer, Berlin, Germany, 1980.

[16] P. Cvitanović, Ed., Universality in Chaos, Adam Hilger, Bristol, UK, 2nd edition, 1989.

[17] M. J. Feigenbaum, "Quantitative universality for a class of nonlinear transformations," Journal of Statistical Physics, vol. 19 , no. 1 , pp. 25-52, 1978.

[18] M. Hénon, "A two-dimensional mapping with a strange attractor," Communications in Mathematical Physics, vol. 50, no. 1, pp. 69-77, 1976.

[19] B. B. Mandelbrot, Fractals: Form, Chance and Dimension, W. H. Freeman, San Francisco, Calif, USA, 1977.

[20] E. N. Lorenz, "Deterministic nonperiodic flow," Journal of the Atmospheric Sciences, vol. 20, pp. 130-141, 1963.

[21] M. Gidea, F. Deppe, and G. Anderson, "Phase space reconstruction in the restricted three-body problem," in New Trends in Astrodynamics and Applications III, E. Belbruno, Ed., vol. 886 of AIP Conference Proceedings, pp. 139-152, 2007.

[22] H. Poincaré, "On the problem of three bodies and the equations of dynamics," Acta Mathematica, vol. 13, pp. 1-270, 1890.
[23] W. Nagourney, J. Sandberg, and H. Dehmelt, "Shelved optical electron amplifier: Observation of quantum jumps," Physical Review Letters, vol. 56, no. 26, pp. 2797-2799, 1986.

[24] J. C. Bergquist, R. G. Hulet, W. M. Itano, and D. J. Wineland, "Observation of quantum jumps in a single atom," Physical Review Letters, vol. 57, no. 14, pp. 1699-1702, 1986. 

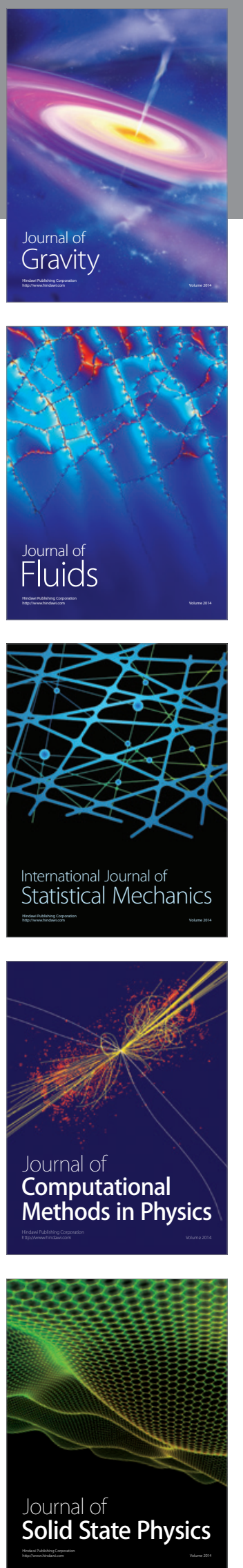

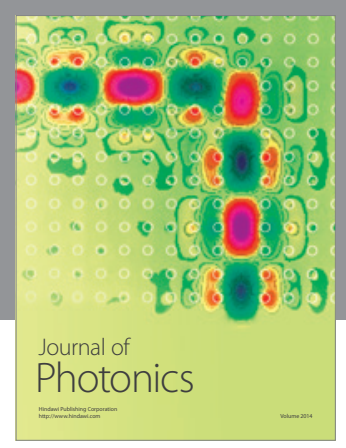

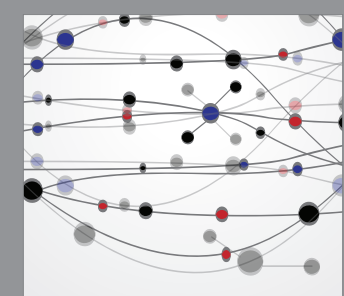

The Scientific World Journal
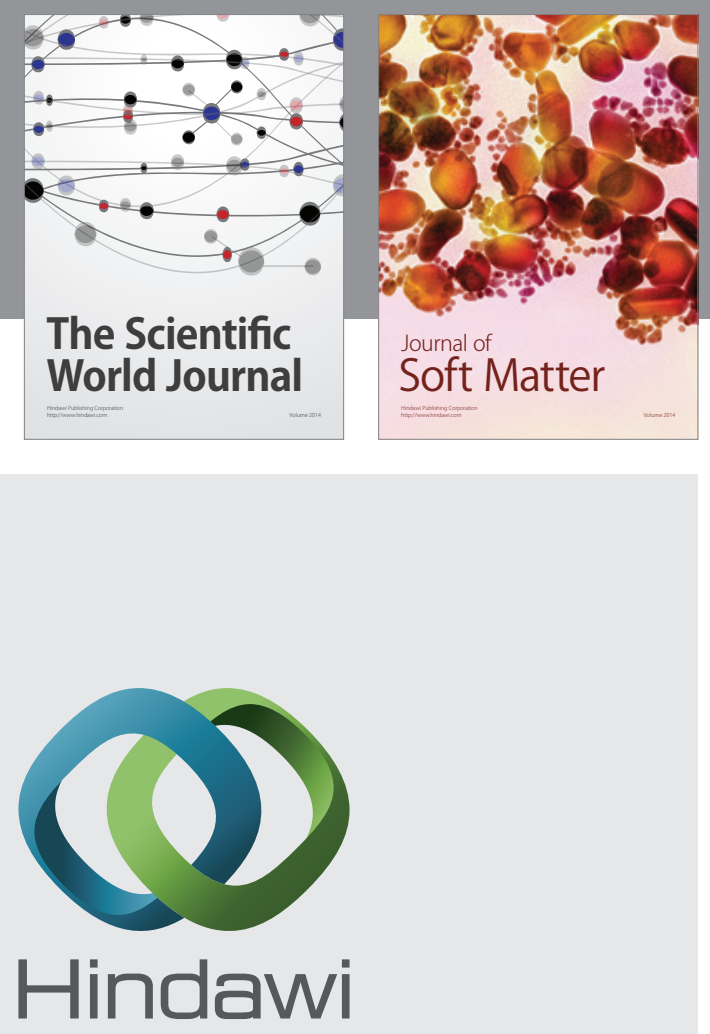

Submit your manuscripts at

http://www.hindawi.com
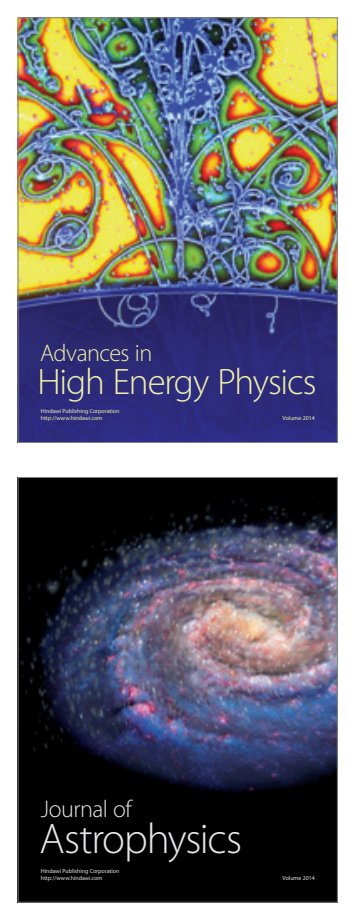
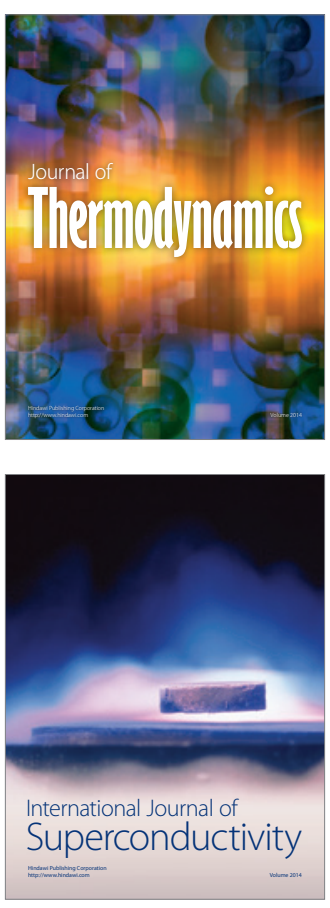
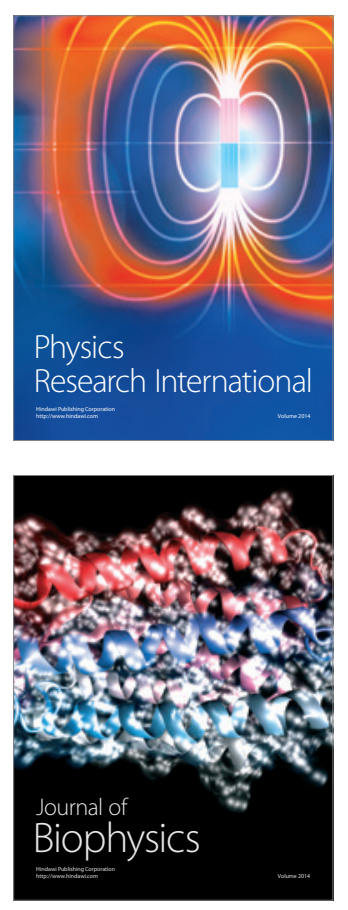
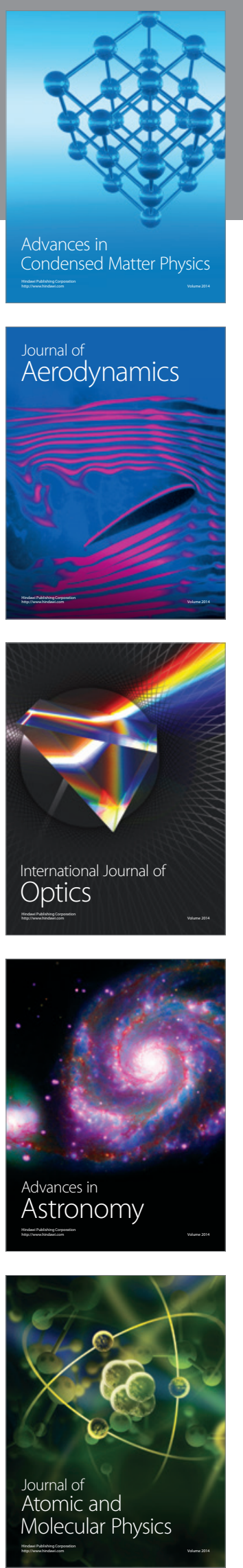